\title{
Supporting Information for Scalable Synthesis of InAs Quantum Dots Mediated through Indium Redox Chemistry
}

\author{
Matthias Ginterseder, Daniel Franke, Collin F. Perkinson, Lili Wang, Eric C. Hansen and \\ Moungi G. Bawendi*
}

*Corresponding author:mgb@mit.edu

\section{Content}

Experimental Details

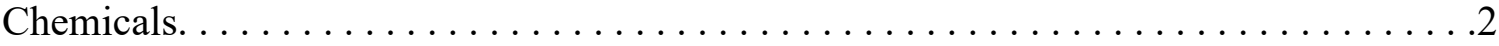

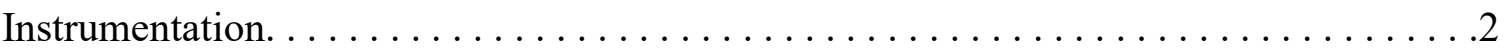

Synthetic procedures. . . . . . . . . . . . . . . . .

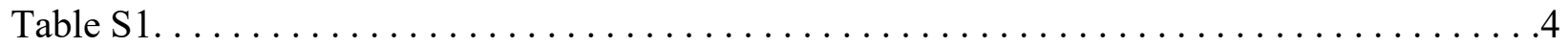

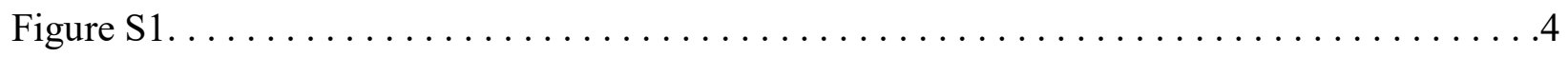

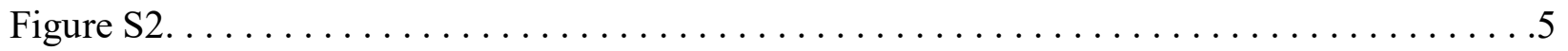

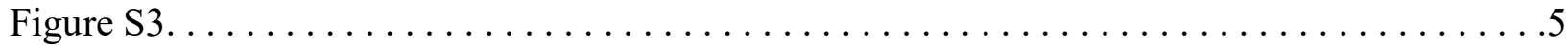

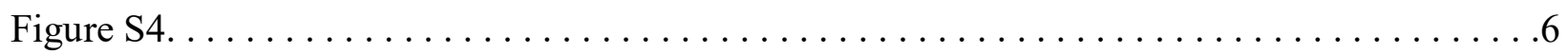

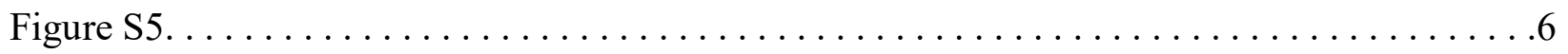

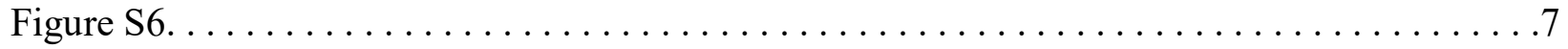

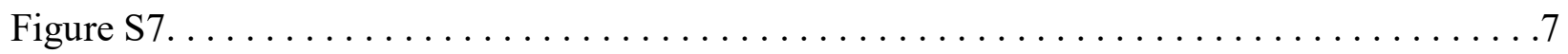

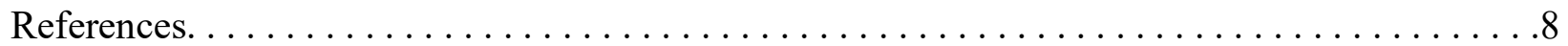




\section{$\underline{\text { Experimental Details }}$}

Chemicals. Indium powder (99.99\%, 325 mesh), indium(I) chloride (InCl, 99.999\%), oleic acid (OA, 90\%), dodecylphosphonic acid (DDPA, 95\%), selenium (99.999\%), and zinc oxide $(\mathrm{ZnO}, 99.999 \%)$ were purchased from Alfa Aesar. Trioctylphosphine (TOP, 97\%), oleylamine (OAm, 70\%), 1-octadecene (ODE, 90\%), toluene (99.8\%), acetonitrile (ACN, 99.9\%), carbon tetrachloride $\left(\mathrm{CCl}_{4}, 99 \%\right)$, and sulfur $(99.998 \%)$ were purchased from Sigma-Aldrich. Indium(III) chloride (InCl $\left.3,99.999 \%\right)$, tris(dimethylamino)arsine (DMAAs, 99\%), and cadmium oxide (CdO, 99.999\%) were purchased from Strem Chemicals. All chemicals were used as received. Indium powder, $\mathrm{InCl}, \mathrm{InCl}_{3}$ and $\mathrm{TOP}$ are stored and handled in an inert atmosphere.

Instrumentation. Absorption spectra were measured on a UV-VIS-NIR spectrometer (Agilent, Cary 5000) and baseline-corrected using absorption through solvents. Photoluminescence (PL) spectra were recorded on a setup built in-house. Following excitation with a $532 \mathrm{~nm}$ diode laser (Thorlabs, CPS532), emission was collected with two silver-coated off-axis parabolic mirrors, focused to the entrance slit of a monochromator (Princeton Instruments, SP2750A) equipped with an $80 \mathrm{gr} / \mathrm{mm}, 1300 \mathrm{~nm} \mathrm{blaze} \mathrm{optical}$ diffraction grating (Princeton Instruments) and imaged using a liquid nitrogen-cooled InGaAs array detector (Princeton Instruments, OMA V:512-1.7 LN). PL quantum yields (PLQYs) were obtained using an integrating sphere (Labsphere, RTC-060SF) setup. The samples were excited with a $785 \mathrm{~nm}$ diode laser (Thorlabs, LDM785) chopped at $210 \mathrm{~Hz}$ using an optical chopper, and the output was recorded with a calibrated germanium photodetector (Newport, 818-IR) using a lock-in amplifier (Stanford Research Systems, SR830). The samples were contained in a PTFE-capped quartz cuvette. A solvent blank was used in conjunction with a colored glass longpass filter (Schott Glass, RG850) blocking the excitation beam to allow correction of the obtained PLQY values for leakage of the excitation light and transmittance of the filter. Additionally, the measured photocurrent was adjusted for the external quantum efficiency of the photodetector. Transmission electron microscopy (TEM) images were acquired on a JOEL 2010 Advanced High Performance TEM operating at $200 \mathrm{kV}$ with a lanthanum hexaboride cathode. Samples were prepared by drop-casting a solution containing the QDs of interest in an apolar solvent (toluene or $\mathrm{CCl}_{4}$ ) onto a copper grid with an amorphous carbon film (Ted Pella, 400 mesh). Powder X-ray diffraction patterns were obtained using a multipurpose diffractometer (Rigaku $\mathrm{Smartlab)}$ with a $9 \mathrm{~kW}$ rotating $\mathrm{Cu}-\mathrm{K} \alpha \mathrm{X}$-ray source aligned in a Bragg-Brentano geometry. For sample preparation, a solution of InAs QDs in toluene was dropcast onto a zero diffraction plate (MTI Corp., Si crystal, P-type B-doped) and the solvent allowed to evaporate. The resulting thin film was encapsulated with polyimide tape to ensure stability of the InAs QDs over the course of the measurement.

\section{Synthetic procedures.}

Standard InAs core synthesis. In a typical synthesis, a $25 \mathrm{~mL}$ three-necked round-bottom flask is equipped with a reflux condenser and charged with $7.2 \mathrm{~mL} \mathrm{OAm}$ and a magnetic stir bar. The solvent is degassed at $100{ }^{\circ} \mathrm{C}(100 \mathrm{mTorr})$ for $1 \mathrm{~h}$. After switching to a nitrogen atmosphere and cooling to room temperature, $0.3 \mathrm{~mL}$ TOP and $75 \mathrm{mg}(0.5 \mathrm{mmol}, 3.1 \mathrm{eq}) \mathrm{InCl}$ are added. Addition of $20 \mathrm{mg}$ DDPA at this point is necessary to achieve core sizes with absorption features at $800 \mathrm{~nm}$ or below. Subsequently, the mixture is heated to $240{ }^{\circ} \mathrm{C}$. Separately, $1 \mathrm{~mL}$ OAm is heated to $50{ }^{\circ} \mathrm{C}$ inside a glove box and $75 \mu \mathrm{L}$ DMAAs are added. After $5 \mathrm{~min}$, gas formation has ceased and $0.4 \mathrm{~mL}(0.16 \mathrm{mmol} \mathrm{As}, 1 \mathrm{eq})$ of the resulting precursor solution are withdrawn with a syringe for injection. Once the reaction mixture reaches reaction temperature, the arsenic precursor is swiftly injected under vigorous stirring. The progress of the reaction is followed by drawing aliquots and measuring their absorption at $450 \mathrm{~nm} \mathrm{in} \mathrm{CCl}_{4}$. After 90 min of reaction time, the flask is cooled down to room temperature and $7.5 \mathrm{~mL}$ toluene, followed by $23 \mathrm{~mL} \mathrm{ACN}$, are added to induce precipitation. The mixture is centrifuged at $8000 \mathrm{rpm}$ for $2 \mathrm{~min}$ and the supernatant discarded. The pellet is redispersed in $5 \mathrm{~mL}$ toluene and crashed one more time with $15 \mathrm{~mL}$ ACN. Following centrifugation, the pellet is redispersed in toluene or hexanes. A last round of centrifugation and filtration through a $0.2 \mu \mathrm{m}$ PTFE syringe filter (VWR Syringe Filters) furnishes the final InAs QD dispersion.

InAs core scale-up. The scaled-up synthesis yielding InAs QDs on gram scale was conducted in an analogous fashion, employing a mechanical stirrer instead of a magnetic stir bar.

InAs core precursor variation. The control experiment employing In and $\mathrm{InCl}_{3}$ as precursors was conducted in an analogous fashion, substituting $75 \mathrm{mg} \mathrm{InCl}$ for $38 \mathrm{mg}$ indium powder and $37 \mathrm{mg} \mathrm{InCl}_{3}$.

InAs core synthesis with hot and continuous injection. This scheme is overall similar to the standard InAs core synthesis above, with some adaptations. In a typical synthesis, a $25 \mathrm{~mL}$ three-necked round-bottom flask is equipped with a reflux condenser and charged with $7.2 \mathrm{~mL}$ OAm and a magnetic stir bar. The solvent is degassed at $100{ }^{\circ} \mathrm{C}(100 \mathrm{mTorr})$ for $1 \mathrm{~h}$. After switching to a nitrogen atmosphere and cooling to room temperature, $0.3 \mathrm{~mL}$ TOP and $225 \mathrm{mg}(1.5 \mathrm{mmol}, 9.3 \mathrm{eq}) \mathrm{InCl}$ are added, and the mixture is heated to $240{ }^{\circ} \mathrm{C}$. Separately, $2 \mathrm{~mL}$ OAm is heated to $50{ }^{\circ} \mathrm{C}$ inside a glove box and $150 \mu \mathrm{L}$ DMAAs are added. After $5 \mathrm{~min}$, gas formation has ceased. $0.4 \mathrm{~mL}(0.16 \mathrm{mmol} \mathrm{As}, 1 \mathrm{eq})$ of the precursor solution are withdrawn for hot injection, and $0.8 \mathrm{~mL}(0.32 \mathrm{mmol} \mathrm{As}, 2 \mathrm{eq})$ are withdrawn in a separate syringe for continuous injection. Once the reaction mixture reaches reaction temperature, the arsenic precursor is swiftly injected under vigorous stirring. After 20 min of reaction time, additional As 
precursor is injected via a syringe pump over $2 \mathrm{~h}$, followed by $30 \mathrm{~min}$ of annealing at $280^{\circ} \mathrm{C}$. The workup is conducted analogously to the standard InAs core synthesis.

InAs core synthesis solely with continuous injection. This scheme closely follows the standard core synthesis up to single hot injection. Instead of the single hot injection, the As precursor is injected into the reaction mixture continuously via a syringe pump over the course of $90 \mathrm{~min}$.

Cadmium oleate $\left(\mathbf{C d}(\mathbf{O l})_{2}\right)$. A $100 \mathrm{~mL}$ three-necked round-bottom flask is charged with $\mathrm{CdO}(1.027 \mathrm{~g}, 8 \mathrm{mmol}, 1 \mathrm{eq})$ and OA $(20$ $\mathrm{mL}, 64 \mathrm{mmol}, 8 \mathrm{eq}$ ). While stirring, the mixture is degassed at room temperature for $1 \mathrm{~h}$, followed by another $2 \mathrm{~h}$ at $100{ }^{\circ} \mathrm{C}$. Subsequently, the flask is switched to a nitrogen atmosphere and heated to $200{ }^{\circ} \mathrm{C}$ until a clear solution is obtained. ODE (20 mL) is added, and the solution is degassed again at $100{ }^{\circ} \mathrm{C}$ for $30 \mathrm{~min}$ to yield a $0.2 \mathrm{M}$ solution of $\mathrm{Cd}(\mathrm{Ol})_{2}$.

Zinc oleate $\left(\mathrm{Zn}(\mathbf{O l})_{2}\right)$. A $0.1 \mathrm{M}$ solution of $\mathrm{Zn}(\mathrm{Ol})_{2}$ is obtained in an analogous fashion to the $\mathrm{Cd}(\mathrm{Ol})_{2}$ solution described above by substituting $\mathrm{CdO}$ for $\mathrm{ZnO}$ and adding $40 \mathrm{~mL}$ of OAm after cooling down to room temperature.

Trioctylphosphine selenide (TOPSe). A $40 \mathrm{~mL}$ scintillation vial is charged with selenium powder (32 mg, $4 \mathrm{mmol}, 1 \mathrm{eq}$ ), TOP $(4 \mathrm{~mL}, 9 \mathrm{mmol}, 2.25 \mathrm{eq})$, and $\mathrm{ODE}(16 \mathrm{~mL})$. The mixture is sonicated until all solids are dissolved to yield a $0.2 \mathrm{M}$ solution of TOPSe.

Sulfur in 1-octadecene (ODE/S). A $100 \mathrm{~mL}$ three-necked round-bottom flask is charged with ODE (40 mL) and sulfur (58 mg, $1.8 \mathrm{mmol}$ ) and stirred while heating to $100{ }^{\circ} \mathrm{C}$ under vacuum until all solids have dissolved, furnishing a $0.045 \mathrm{M}$ solution of $\mathrm{ODE} / \mathrm{S}$

General shell growth remarks. For all shell growth procedures, the amount of precursors necessary to grow a certain shell thickness is estimated by a sizing curve based on spherical particles. ${ }^{1}$ In short, an absorption spectrum of a purified InAs QD solution is recorded, and the position of the first excitonic feature, together with the absorption at $450 \mathrm{~nm}$, are used to assess the size and concentration of QDs in solution. Precursor amounts are then determined based on the material needed to grow successive monolayers on cores of the specified size. In case of more than one shell, the estimated amounts of precursor required for the second shell are treated like a continuation of the first shell. The progress of shell growth was tracked by drawing aliquots and measuring absorption, emission lineshape, and PLQY dispersed in $\mathrm{CCl}_{4}$.

CdSe shell growth. In a typical reaction, a $50 \mathrm{~mL}$ four-necked round-bottom flask is charged with OAm (5 mL) and ODE (5 mL), connected to a condenser and degassed at $100{ }^{\circ} \mathrm{C}$ for $1 \mathrm{~h}$ under stirring. InAs QDs (40 nmol, $\left.4.2 \mathrm{~nm}\right)$ in hexanes, synthesized and purified as described above, are introduced and the volatiles removed by degassing at room temperature for $1 \mathrm{~h}$. Separately, two syringes with $0.05 \mathrm{M} \mathrm{Cd}(\mathrm{Ol})_{2}(1.75 \mathrm{~mL}, 88 \mu \mathrm{mol})$ and $0.05 \mathrm{M}$ TOPSe $(1.75 \mathrm{~mL}, 88 \mu \mathrm{mol})$ in ODE are prepared. The InAs dispersion is heated to $240{ }^{\circ} \mathrm{C}$ for shell growth. At $100{ }^{\circ} \mathrm{C}$, the atmosphere is switched to nitrogen, and at $200{ }^{\circ} \mathrm{C}$, injection of the two shell precursor solutions is started at a rate of $0.9 \mathrm{~mL} / \mathrm{h}$. After completion of the shell growth, the core-shell particles can be purified by addition of toluene and precipitation with ACN twice. The exact amounts needed will vary based on initial core size and thickness of the shell grown. The final particles are filtrated through a $0.2 \mu \mathrm{m}$ syringe filter and stored in toluene or hexanes.

CdS and ZnS shell growth. Overcoating of InAs/CdSe core-shell QDs with a second shell of either CdS or ZnS is carried out analogously to the CdSe shell growth. For $\mathrm{ZnS}, 0.05 \mathrm{M} \mathrm{Cd}(\mathrm{Ol})_{2}$ is substituted with $0.05 \mathrm{M} \mathrm{Zn}(\mathrm{Ol})_{2}$ in ODE. Both procedures use $0.045 \mathrm{M}$ ODE/S in ODE as the chalcogenide precursor and a $10 \%$ excess of $\mathrm{Cd} / \mathrm{Zn}$ compared to $\mathrm{S}$, respectively. 
Table S1. Overview of reaction conditions and resultant InAs core optical absorption properties.

\begin{tabular}{|c|c|c|c|}
\hline Temperature $/{ }^{\circ} \mathrm{C}$ & Additive & Reaction Time / min & First abs. feature $/ \mathrm{nm}$ \\
\hline 210 & $20 \mathrm{mg}$ DDPA & 30 & 704 \\
210 & $20 \mathrm{mg}$ DDPA & 60 & 757 \\
210 & - & 30 & 801 \\
210 & - & 90 & 855 \\
240 & - & 90 & 938 \\
270 & - & 30 & 964 \\
300 & - & 2 & 968 \\
300 & - & 30 & 1051 \\
310 & - & 10 & 1092 \\
310 & - & 20 & 1139 \\
\hline
\end{tabular}

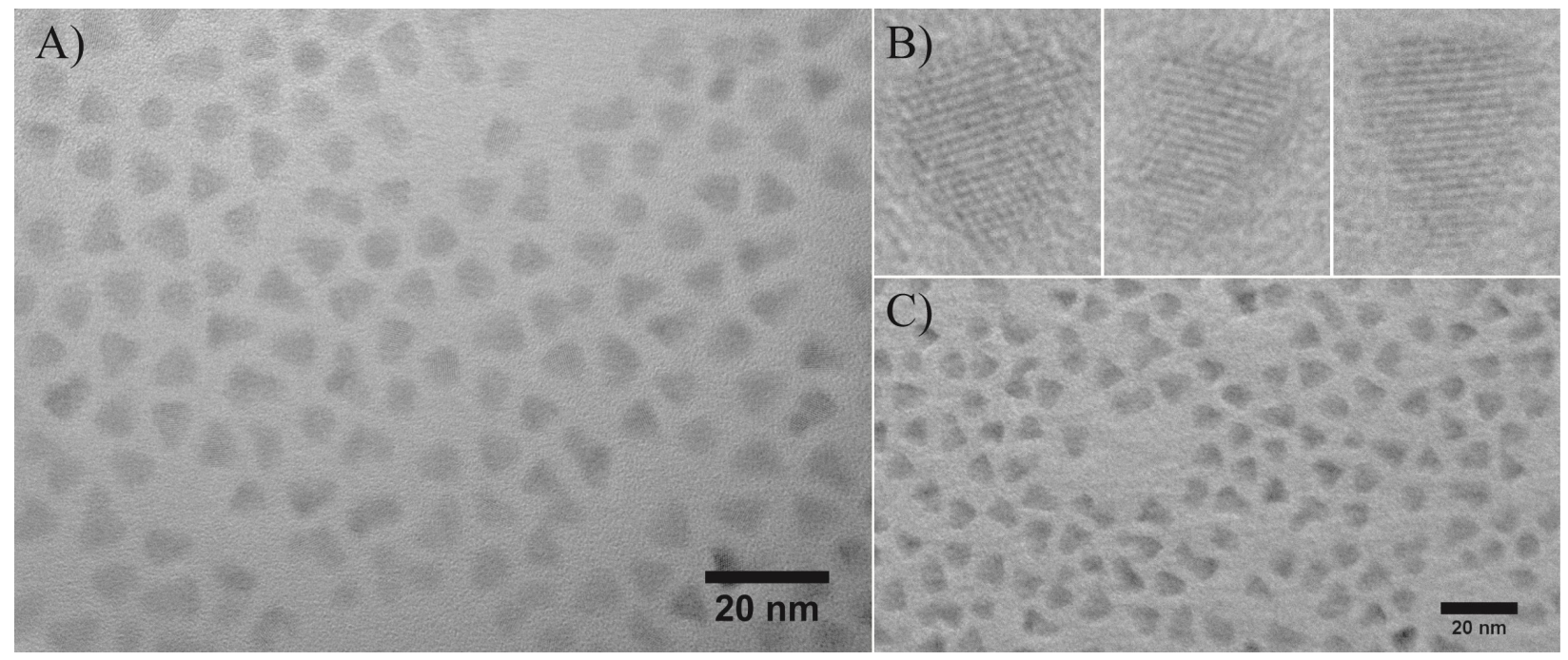

Figure S1. Additional HR-TEM images of InAs QDs synthesized at $320{ }^{\circ} \mathrm{C}$. A, C) Representative HR-TEM image with B) magnified individual QDs from A) exhibiting good crystallinity throughout the particles. 


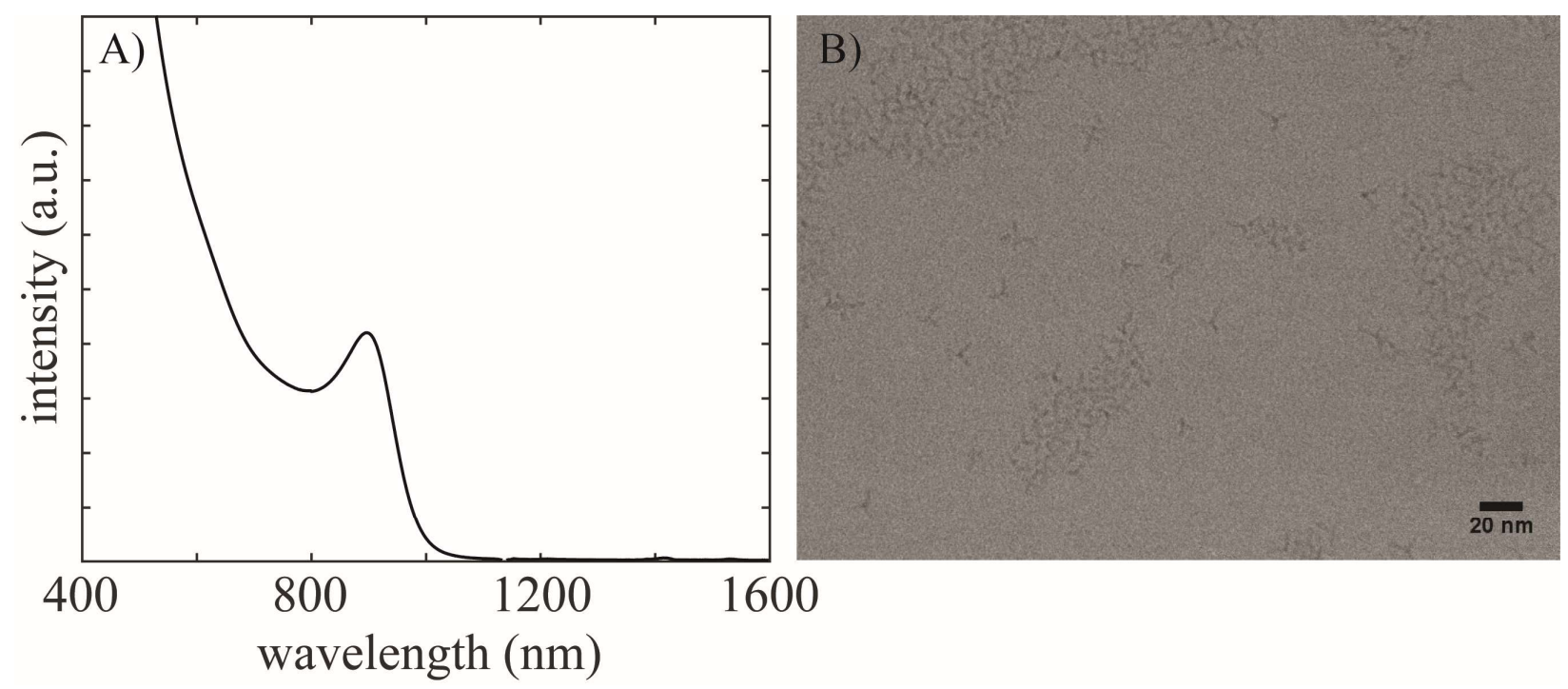

Figure S2. InAs tetrapods grown at early times and low temperature during InAs core synthesis. At early times and intermediate temperatures, InAs QDs can appear tetrapodal. A) Absorption curve of an aliquot withdrawn after 10 min of a standard synthesis at $240{ }^{\circ} \mathrm{C}$. B) Corresponding TEM image exhibiting anisotropic QD growth.
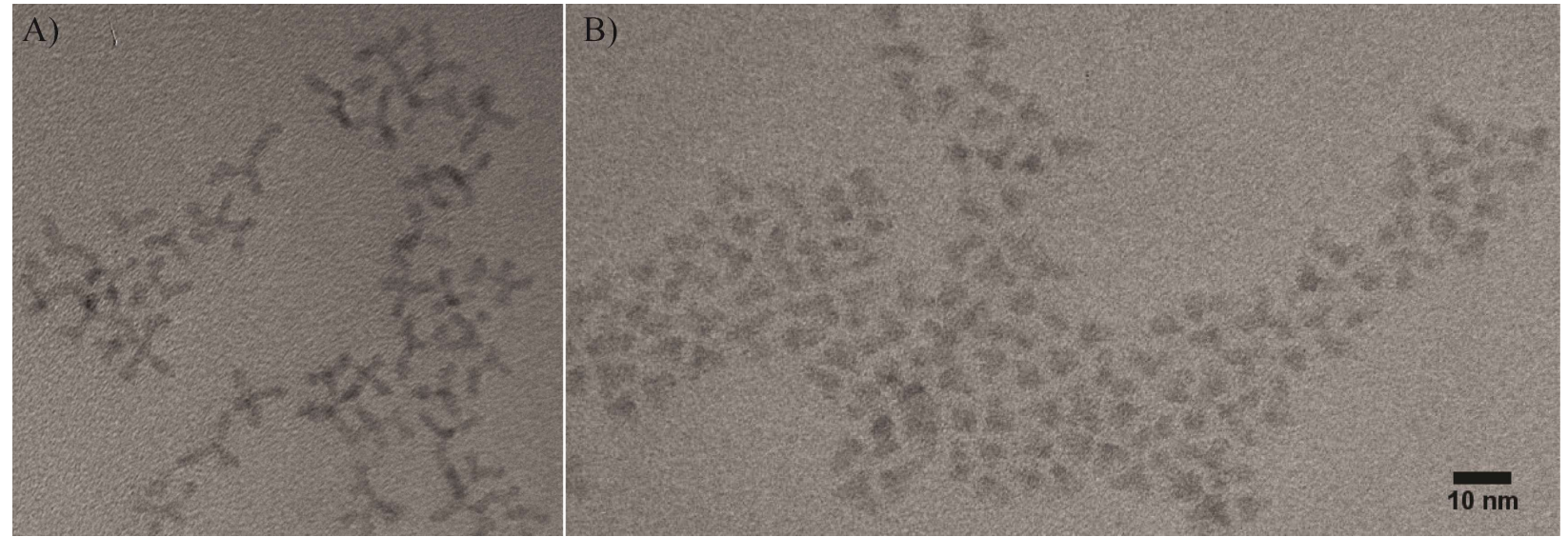

Figure S3. Annealing of tetrapodal InAs QDs. A) Tetrapodal QDs can be annealed to reach B) more spherical shape. 


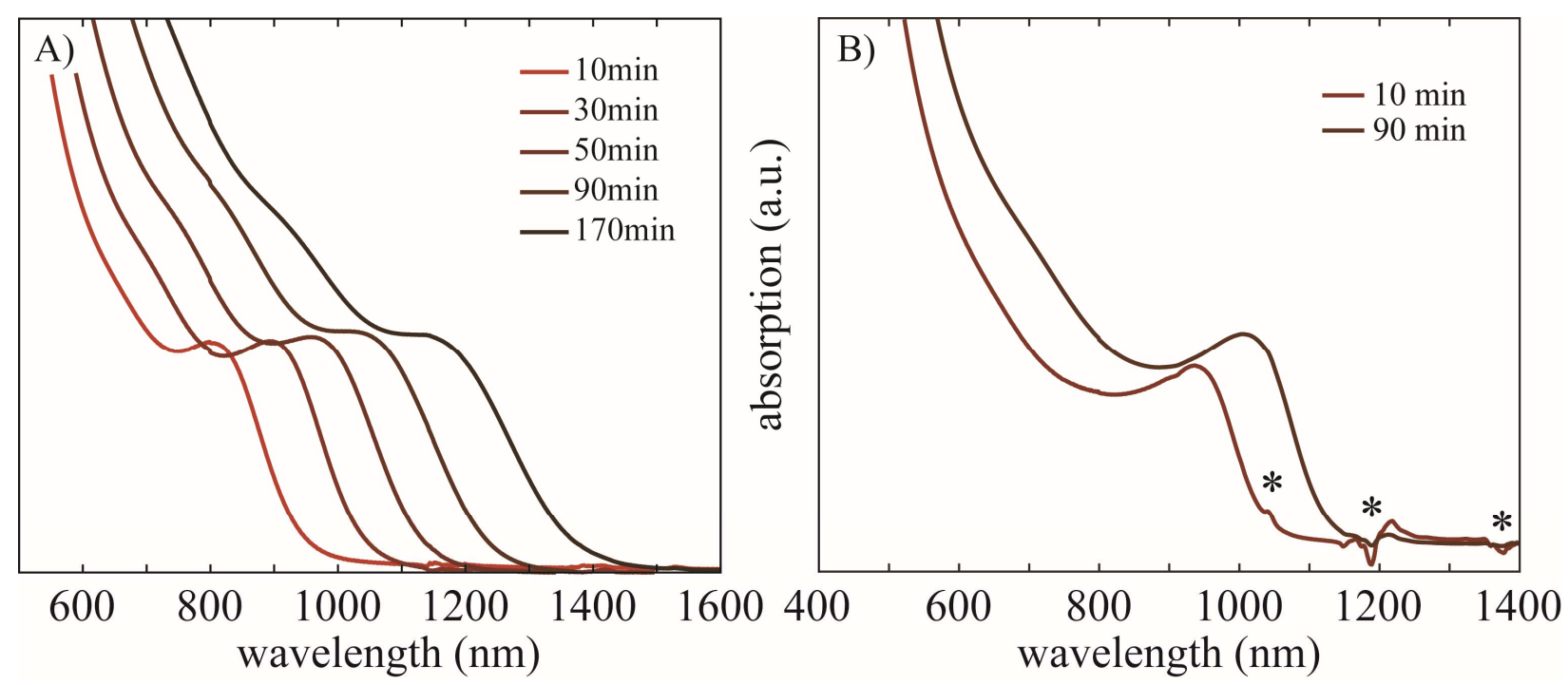

Figure S4. Continuous injection synthesis. A) Absorption curves for a synthesis conducted with a combination of hot and continuous injection. Sustained growth of the formed InAs cores is observed. B) Absorption curves of a synthesis conducted solely by continuous injection of As precursor. The asterisks indicate residual solvent signals.
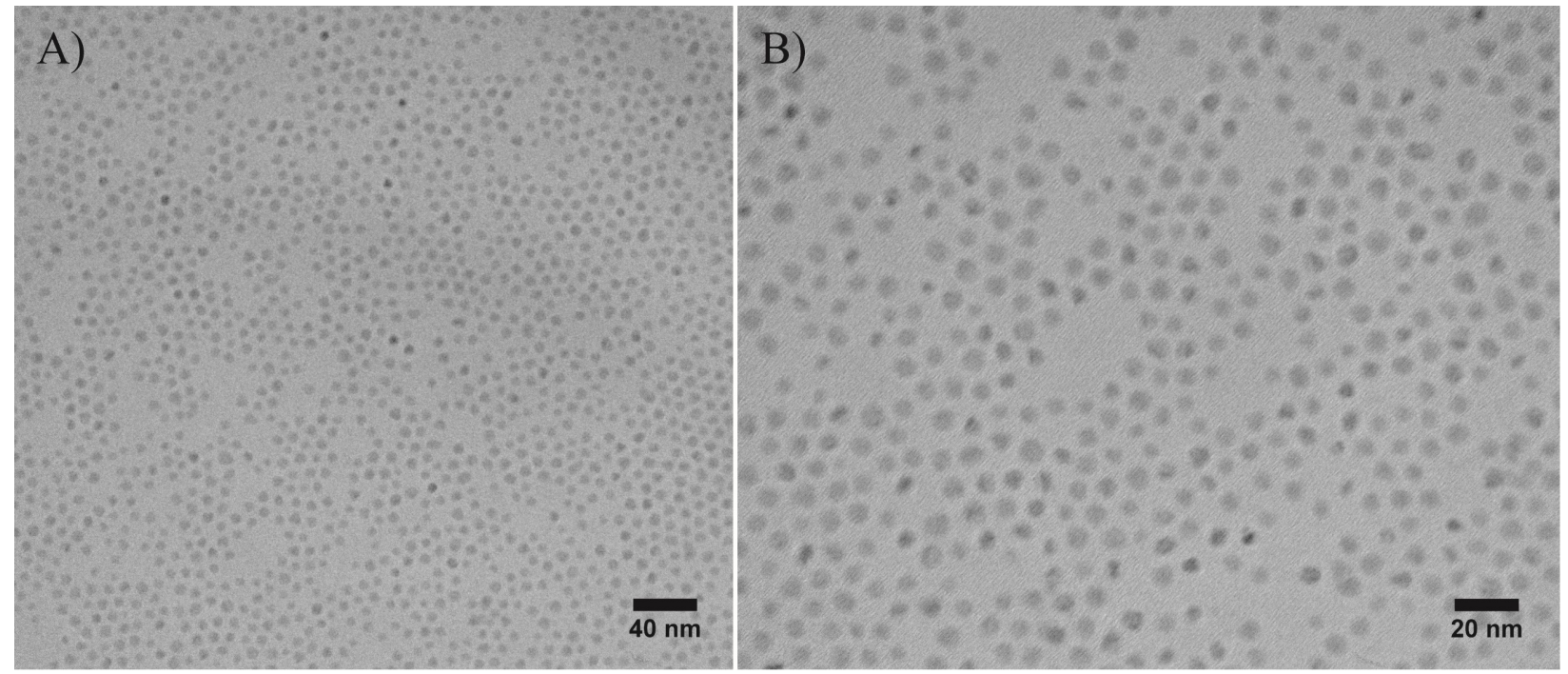

Figure S5. Additional images of InAs/CdSe core-shell QDs. A, B) Representative HR-TEM images of core-shell QDs. 

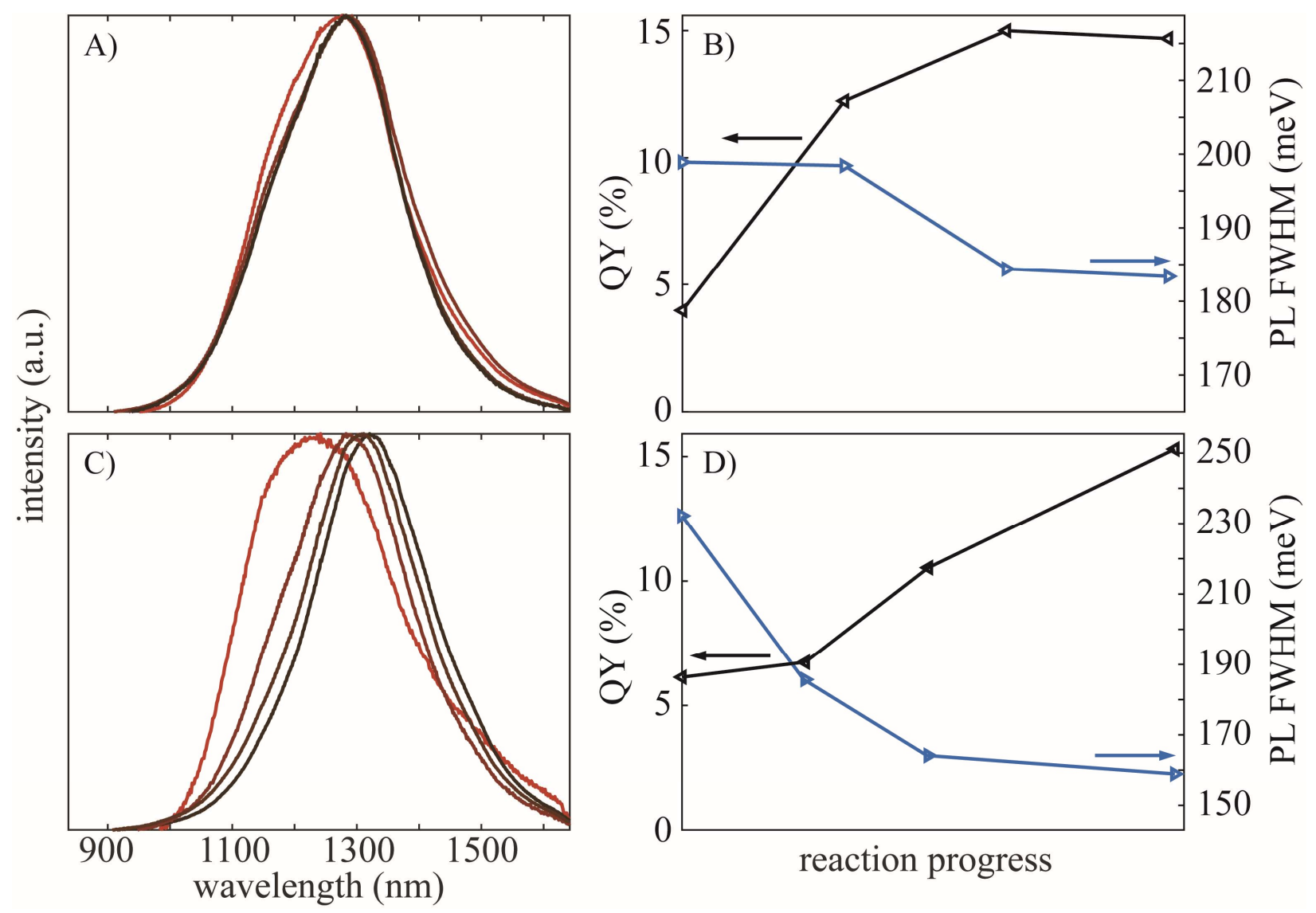

Figure S6. InAs/CdSe/CdS core-shell-shell growth. A, C) Typical progression of emission lineshape for CdS shell growth on top of InAs/CdSe QDs. B, D) Corresponding progression of QY and PL FWHM over the course of the shelling.
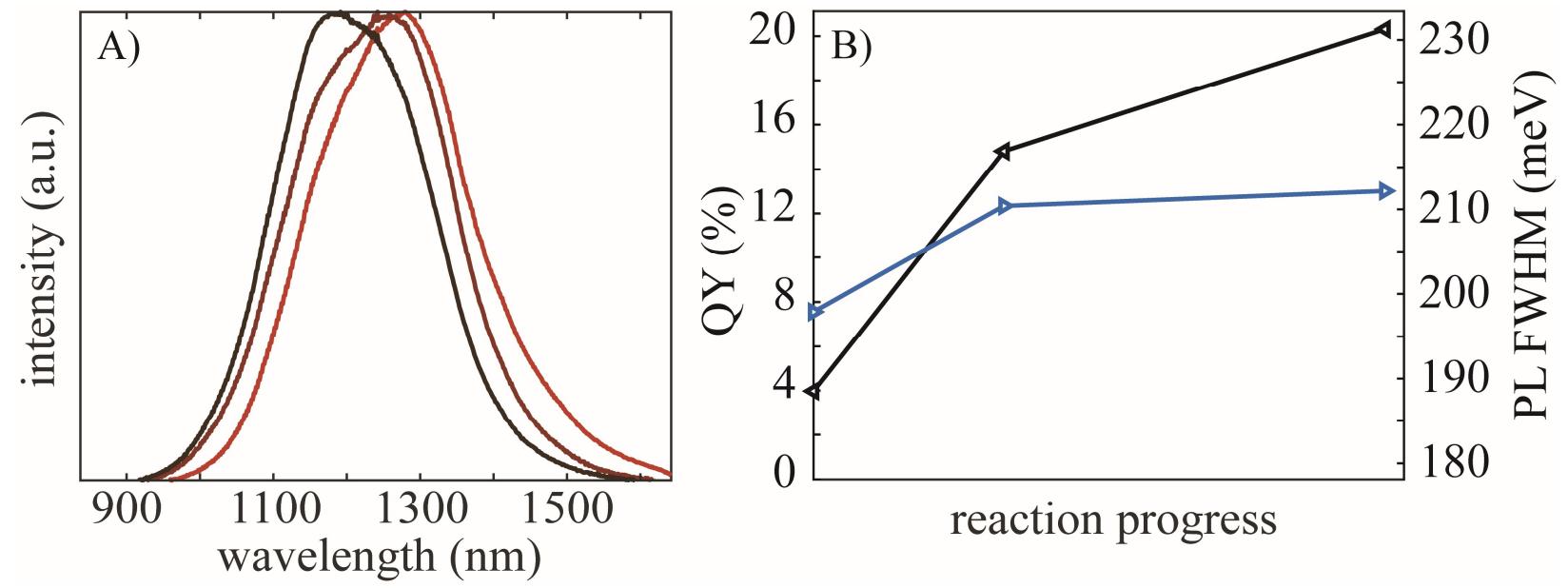

Figure S7. InAs/CdSe/ZnS core-shell-shell growth. A) Typical progression of emission lineshape for ZnS shell growth on top of InAs/CdSe QDs. B) Corresponding progression of QY and PL FWHM over the course of the shelling. 
$\underline{\text { Reference }}$

(1) Yu, P.; Beard, M. C.; Ellingson, R. J.; Ferrere, S.; Curtis, C.; Drexler, J.; Luiszer, F.; Nozik, A. J. Absorption Cross-Section and Related Optical Properties of Colloidal InAs Quantum Dots. J. Phys. Chem. B 2005, 109 (15), 7084-7087. 